\title{
Protein localization in electron micrographs using fluorescence nanoscopy
}

\author{
Shigeki Watanabe ${ }^{1}$, Annedore Punge $^{2}$, Gunther Hollopeter ${ }^{1}$, Katrin I Willig ${ }^{2}$, Robert John Hobson ${ }^{1}$, \\ M Wayne Davis ${ }^{1}$, Stefan W Hell ${ }^{2} \&$ Erik M Jorgensen ${ }^{1}$
}

\begin{abstract}
A complete portrait of a cell requires a detailed description of its molecular topography: proteins must be linked to particular organelles. Immunocytochemical electron microscopy can reveal locations of proteins with nanometer resolution but is limited by the quality of fixation, the paucity of antibodies and the inaccessibility of antigens. Here we describe correlative fluorescence electron microscopy for the nanoscopic localization of proteins in electron micrographs. We tagged proteins with the fluorescent proteins Citrine or tdEos and expressed them in Caenorhabditis elegans, fixed the worms and embedded them in plastic. We imaged the tagged proteins from ultrathin sections using stimulated emission depletion (STED) microscopy or photoactivated localization microscopy (PALM). Fluorescence correlated with organelles imaged in electron micrographs from the same sections. We used these methods to localize histones, a mitochondrial protein and a presynaptic dense projection protein in electron micrographs.
\end{abstract}

Proteins can be imaged in cells by tagging them with fluorescent proteins or antibodies. However, the resolution of conventional optical approaches is limited to about $200 \mathrm{~nm}$ by the diffraction of light and to even poorer resolutions in practice ${ }^{1}$. By contrast, typical proteins are about $4 \mathrm{~nm}$ in diameter and may be associated with organelles as small as $30 \mathrm{~nm}$. Thus, localization of proteins to cellular structures using fluorescence methods is fairly crude. Recently, fluorescence techniques capable of nanometer-scale resolution ('nanoscopy') ${ }^{2}$ have been developed, which permit separation of fluorophores closer than the diffraction limit ${ }^{2}$.

In stimulated emission depletion (STED) microscopy ${ }^{3}$, fluorescence is inhibited by a beam of light, called the STED beam. Patterned as a doughnut and overlaid with the excitation beam of a scanning microscope, this beam ensures that only fluorophores in a narrow region around the doughnut center are allowed to fluoresce; the other molecules illuminated by the excitation light remain dark ${ }^{4}$. Photoactivated localization microscopy (PALM) and similar techniques (fluorescence PALM (FPALM) and stochastic optical reconstruction microscopy (STORM) $)^{5-7}$ use photoactivatable molecules whose fluorescence is activated by the absorption of a photon (usually ultraviolet). To separate features that are closer than the diffraction limit, only one fluorophore is randomly activated; the neighboring molecules remain dark. The position of the fluorophore is determined by calculating the centroid of the emission pattern. The registered molecules are subsequently turned off by bleaching, allowing adjacent molecules to be activated and become fluorescent. This sequence is continued until all molecules are registered.

Fluorescence nanoscopy can be used to localize proteins precisely ${ }^{5}$, but the cellular context is limited in these images. Immunocytochemical electron microscopy (immuno-EM) can be used to localize proteins to organelles. However, this method is compromised by technical difficulties including the destruction of antigens, inaccessibility of antigens, the lack of suitable antibodies and nonspecific binding of antibodies ${ }^{8-10}$. Even when this method is used successfully, the size of antibodies $(\sim 19 \mathrm{~nm} \text { long })^{11}$ limits the ultimate resolution, particularly when secondary antibodies are used.

The advantage of fluorescence microscopy is that all proteins can potentially be tagged with a fluorophore. The advantage of electron microscopy is its exquisite depiction of subcellular structure. As their strengths are complementary, these two methods can be very effective if combined ${ }^{12,13}$. PALM has been performed on cryo-sectioned material fixed with aldehydes ${ }^{5}$. However, subcellular details are obscure in cryo-sections because of poor tissue contrast. Morphology could be improved using traditional electron microscopy techniques, but fluorescent proteins are quenched by the acidic, dehydrated and oxidizing conditions required for fixation and polymer embedding of the specimen ${ }^{14}$. Ultimately, a compromise must be found between preservation of fluorescence and morphology. To develop a practical method for correlative fluorescence electron microscopy, here we optimized each step of sample preparation, balancing the requirements for fluorescence and ultrastructure. As our model system, we used the nematode Caenorhabditis elegans because fluorescently tagged proteins can be stably expressed ${ }^{15,16}$ and methods for electron microscopy are well established for this organism ${ }^{9}$. We developed methods to use both STED microscopy and PALM on ultrathin sections of fixed tissues to localize proteins at the nanoscale and subsequently correlate protein localization with ultrastructural features revealed by electron microscopy.

${ }^{1}$ Department of Biology and Howard Hughes Medical Institute, University of Utah, Salt Lake City, Utah, USA. ${ }^{2}$ Department of NanoBiophotonics, Max Planck Institute for Biophysical Chemistry, Göttingen, Germany. Correspondence should be addressed to E.M.J. (jorgensen@biology.utah.edu).

RECEIVED 3 JUNE; ACCEPTED 20 OCTOBER; PUBLISHED ONLINE 21 NOVEMBER 2010; DOI:10.1038/NMETH.1537 

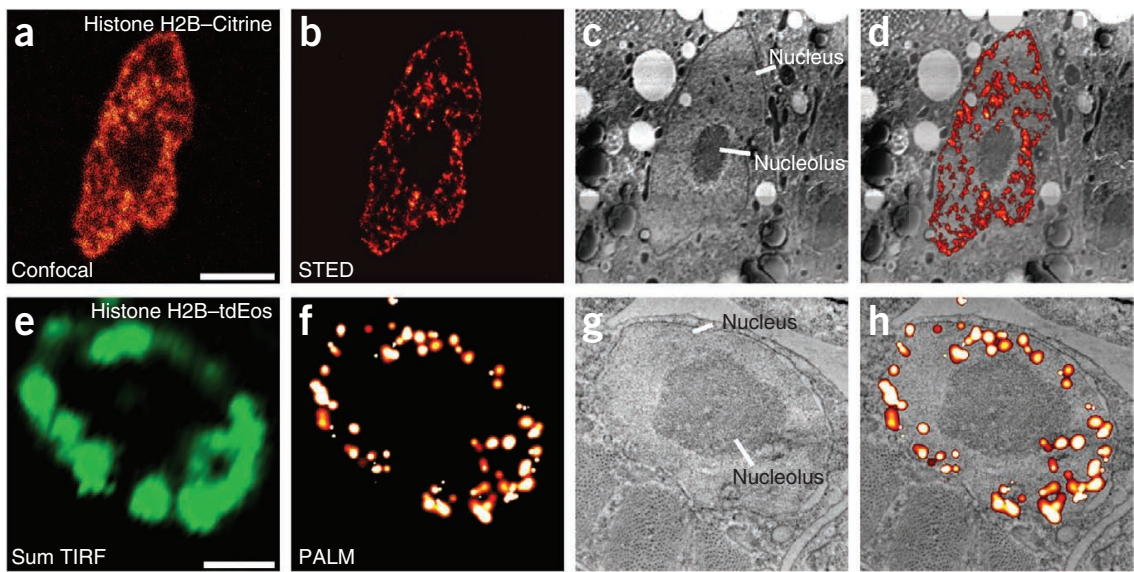

Figure 1 | Correlative fluorescence and electron microscopy using histone H2B fusion proteins. (a-c) Confocal image (a), STED image (b) and electron micrograph (c) from the same thin GMA section $(120 \mathrm{~nm}$ ) from a worm expressing histone H2B-Citrine. (d) Correlative STED microscopy and electron micrographs showing histone $\mathrm{H} 2 \mathrm{~B}$-Citrine (overlay of the images in $\mathbf{b}$ and $\mathbf{c}$ ). The images in a-d show an intestinal cell nucleus. (e-g) Sum TIRF image (e; represents all the photons detected by the camera during the experiment), PALM image (f) and electron micrograph (g) from a thin GMA section $(70 \mathrm{~nm}$ ) from a worm expressing histone H2B-tdEos. (h) Correlative PALM and electron micrographs showing histone $\mathrm{H} 2 \mathrm{~B}$-tdEos (overlay of the images in $\mathbf{f}$ and $\mathbf{g}$ ). The images in $\mathbf{e}-\mathbf{h}$ show a muscle cell nucleus. Scale bars, $3 \mu \mathrm{m}(\mathbf{a}-\mathbf{d})$ and $1 \mu \mathrm{m}(\mathbf{e}-\mathbf{h})$.

\section{RESULTS}

\section{Choice of target proteins}

To evaluate fluorescence localization in electron micrographs, we tagged three proteins with well-described localization: histone $\mathrm{H} 2 \mathrm{~B}$ (encoded by his-11), translocase of outer mitochondrial membrane-20 (TOM20; encoded by tomm-20) and $\alpha$-liprin (encoded by syd-2). Histones are localized to the nucleus. The nucleus can be easily visualized using a conventional light microscope, and the use of histone $\mathrm{H} 2 \mathrm{~B}$ is ideal for rapid optimization of preservation of fluorescence (Fig. 1). TOM20 is a $20-\mathrm{kDa}$ mitochondrial outer membrane protein. A cross-section of mitochondria can be as narrow as $150 \mathrm{~nm}$ in diameter, which is below the diffraction limit, and thus TOM20 localization is a good test of super-resolution methodologies (Fig. 2). $\alpha$-liprin is localized to the presynaptic dense projection ${ }^{17}$. Neurons in C. elegans are the most sensitive tissue to fixation and $\alpha$-liprin localization required us to fully optimize our protocol (Fig. 3).

\section{Optimization of fixatives}

Fixatives cross-link cellular structures and protect the tissue from distortions caused by dehydration and embedding in plastic. morphology from ultrathin sections of neurons because of their sensitivity to fixation. As anticipated, fixation with aldehyde-based fixatives (paraformaldehyde, glutaraldehyde and acrolein) resulted in induction of autofluorescence. Autofluorescence could be quenched by $1 \%$ sodium borohydride ${ }^{19}$, but cell membranes were not well preserved by aldehyde-based fixatives (Supplementary Fig. 1a,b). A low concentration of osmium tetroxide $(0.1 \%)$ preserved membrane morphology effectively, but fluorescence was reduced to less than $10 \%$ after fixation and embedding in plastic (Supplementary Fig. 1c,d). If we applied osmium tetroxide at concentrations of less than $0.1 \%$, we observed substantial degradation in tissue morphology. Another lipid cross-linking agent, potassium permanganate ${ }^{9}(0.1 \%)$ preserved membrane morphology well (Supplementary Fig. 1e). Despite the fact that potassium permanganate is also an oxidizing agent, the fluorescence was about 30 -fold brighter than with $0.1 \%$ osmium tetroxide (Supplementary Fig. 1f). However, synaptic vesicles appeared to be missing from the synaptic terminals (Supplementary Fig. 1e). To optimize membrane morphology, we added $0.001 \%$ osmium tetroxide to $0.1 \%$ potassium permanganate. With this cocktail, the morphology of neurons was better than with
Figure 2 | Correlative fluorescence and electron microscopy using TOM20 fusion proteins. (a-c) Confocal image (a), STED image (b) and electron micrograph (c) from the same GMA thin section $(120 \mathrm{~nm})$ of a worm expressing TOM20-Citrine. (d) Correlative STED and electron micrographs showing TOM20-Citrine (overlay of the images in b and $\mathbf{c}$ ). (e-g) Sum TIRF image (e), PALM image (f) and electron micrograph (g) from a thin LR White section $(70 \mathrm{~nm})$ of a worm expressing T0M20-tdEos. (h) Correlative

PALM and electron micrographs showing TOM20-tdEos (overlay of the images in $\mathbf{f}$ and $\mathbf{g}$ ). PALM images of sections from a worm expressing TOM20-tdEos are from tissue embedded in LR White; all other samples were embedded in GMA. Scale bars, $1 \mu \mathrm{m}(\mathbf{a}-\mathbf{d})$ and $2 \mu \mathrm{m}(\mathbf{e}-\mathbf{h})$.
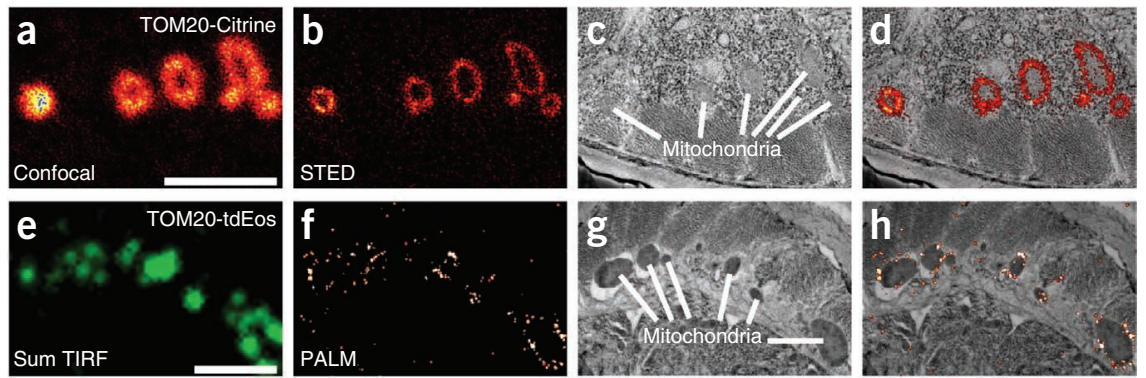
either fixative alone at these concentrations (Supplementary Fig. 1g). Fluorescence was almost as bright as with $0.1 \%$ potassium permanganate alone (fluorescence was $40 \%$ of that for untreated worms; Supplementary Fig. 1h), and we observed strong fluorescence for both Citrine and tdEos after sectioning (Fig. 1a,e).

\section{Optimization of plastic}

After fixation, we embedded tissue samples in plastic resin for ultrathin sectioning. Polymerization typically requires dehydration and heat, which tend to denature proteins, including fluorophores. We processed worms expressing Citrine or tdEos fused to histone H2B (Phsp-16.41:: fluor::his-11::unc-54 3'UTR) as described above and embedded them in the following hydrophilic resins capable of lowtemperature polymerization: Lowicryl K4M (from EMS), LR Gold (from EMS), LR White (from EMS) and glycol methacrylate (GMA; from SPI). Additionally, we included $2-5 \%$ water in each resin. After polymerization, we assessed fluorescence preservation and sectioning quality.

Lowicryl K4M, the most hydrophilic resin, can reportedly tolerate up to $10 \%$ water by weight ${ }^{20}$. However, in our hands, inclusion of $5 \%$ water reduced the sectioning quality of the tissues beyond an acceptable level owing to poor polymerization.

LR Gold and LR White are very similar in their formula and chemistry; however, slightly different problems arose with polymerization. LR Gold polymerized rapidly but did not penetrate the tissue. The $\mathrm{pH}$ of LR White was too acidic for most fluorescent proteins; the $\mathrm{pH}$ with $2-5 \%$ water was quite low $(\sim 5.5)$. Neutralizing the $\mathrm{pH}$ of the plastic using ethanolamine preserved fluorescence (Supplementary Fig. 1) and resulted in good morphology (Supplementary Fig. 1g). However, batch-to-batch variability in $\mathrm{pH}$ and water capacity of LR White resulted in irregular polymerization.

GMA, by contrast, requires $3 \%$ water for polymerization at $\mathrm{pH} 8$, fulfilling both the hydration and alkalinity requirements of the fluorophores. Fluorescence was slightly brighter in GMAembedded samples compared to those in LR White. Moreover, application of water to ultrathin sections collected from worms embedded in GMA immediately increased fluorescence intensity by $30 \%$. This restoration of fluorescence suggests that a large fraction of the fluorescent proteins are maintained in a nonfluorescent, dehydrated state and that about $70 \%$ of the fluorescence observed before fixation can be preserved. Ultrathin sections were difficult to cut because GMA does not cross-link to the cuticle like epoxy resin, and thus the tissue usually breaks loose from the surrounding resin if sectioned below $70 \mathrm{~nm}$. However, we could resolve ultrastructure even on thicker sections by using low accelerating voltage and collecting back-scattered electrons on the scanning electron microscope.

Correlative fluorescence nanoscopy and electron microscopy We imaged proteins tagged with Citrine using STED microscopy (Figs. 1a,b, 2a,b and $\mathbf{3 a}, \mathbf{b}$ ). We embedded transgenic strains expressing Citrine-tagged proteins in GMA and cut $\sim 100$-nm-thick sections to ensure sufficient signal strength. In confocal mode, fluorescence was diffuse, but resolution greatly improved when we applied STED microscopy. Resolution of STED fluorescence in these images was $60-80 \mathrm{~nm}$ by full-width half-maximum analysis of point-like emitters. It was difficult to assign histone $\mathrm{H} 2 \mathrm{~B}$ fluorescence to a particular structure in the nucleus (Fig. 1b), but it was clearly restricted to profiles with a shape similar to that of the nucleus. STED images of samples containing tagged TOM20 revealed circular rings (Fig. 2b). A single fluorescent spot in the confocal image of a sample containing tagged $\alpha$-liprin, resolved into a smaller circle in the STED image but did not reveal a recognizable structure as expected for this very small organelle (Fig. $\mathbf{3 b}$ ). We acquired corresponding scanning electron micrographs from the same sections used for STED imaging. Then we aligned the STED images to the electron micrographs using fluorescent silica beads as fiduciary marks, which we applied onto the sections before fluorescence imaging (Supplementary Fig. 2). The beads are fluorescent in UV light (excitation, $354 \mathrm{~nm}$ and emission, $450 \mathrm{~nm}$ ), and we detected them in an additional imaging scan after recording the STED data. Silica beads become charged and reflect electrons, which resulted in black circles of $1 \mu \mathrm{m}$ in diameter in electron micrographs. The organization of labeled histone $\mathrm{H} 2 \mathrm{~B}$ molecules into chromatin aligned on top of the profile of a nucleus (Fig. 1c,d). The rings of TOM20-Citrine seen in the STED image align with the outer membranes of mitochondria (Fig. 2c,d). The $\alpha$-liprin-Citrine spots observed in the STED images localized to a presynaptic dense projection observed in electron micrographs (Fig. 3c,d and Supplementary Fig. 3a,b).

For PALM, we fused the target proteins to tdEos or Dendra and fixed the transgenic worms under the same conditions as above. Resolution in PALM depends on the number of photons collected from each fluorescent protein, and the localization precision is determined as a function of molecular photon statistics, 
background noise and pixilation ${ }^{21}$. Given the signal-to-noise ratio achieved using Dendra and tdEos, we calculated a localization precision of $12 \mathrm{~nm}$ (Online Methods). Section thickness for PALM was about two-thirds of that used for STED microscopy, and the fluorescence signals were correspondingly reduced compared to STED microscopy. Fluorescence localization was as expected: histone $\mathrm{H} 2 \mathrm{~B}$ fluorescence corresponded to the size of the nucleus and appeared as circles several micrometers in diameter (Fig. 1f). TOM20 signals were confined to rings but were considerably less intense than those observed in the STED image (Fig. 2f). $\alpha$-liprin signals were rare and uninterpretable in the absence of ultrastructural information (Fig. 3f). We correlated PALM fluorescence to the electron micrographs using $100-\mathrm{nm}$ gold nanoparticles as fiduciary markers (Supplementary Fig. 4). Gold nanoparticles are fluorescent ${ }^{22}$; we excited the particles with a $561-\mathrm{nm}$ laser and collected emission at $580 \mathrm{~nm}$ to mark the fluorescence micrographs. Gold particles also reflect the electron beam in the scanning electron microscope and mark the micrograph. In the alignments, tagged histones localized to the nucleus but not the nucleolus (Fig. 1g,h). The distribution of histone H2B in the nucleus of a muscle cell was confined to the rim of the inner membrane compared to the distribution of histone $\mathrm{H} 2 \mathrm{~B}$ in the intestinal cell, but these differences are likely due to the cell type rather than the technique (Supplementary Fig. 5). TOM20 molecules were localized to the outer membrane of mitochondria (Fig. 2g,h). Tagged $\alpha$-liprin was expressed from multicopy arrays for both STED microscopy and PALM. Overexpression of liprin resulted in aggregations of the protein in the cell body adjacent to the nucleus (Fig. $3 \mathbf{h}$ and Supplementary Fig. 3c,d). $\alpha$-liprintdEos signals at the synapse were localized to the presynaptic dense projection (Fig. $\mathbf{3 g}, \mathbf{h}$ ).

\section{DISCUSSION}

For correlative fluorescence electron microscopy, scanning electron microscopy has several advantages over transmission electron microscopy. First, sections for scanning electron microscopy can be mounted on a coverglass, which allows hundreds of sections in long ribbons to be examined for fluorescence, which can later be assembled for array tomography ${ }^{13}$. Moreover, PALM is performed in the total internal reflection fluorescence (TIRF) configuration, which requires mounting on a coverglass. Second, thick sections can be imaged with high resolution in scanning but not transmission electron microscopy. Sections must be relatively thick $(70-100 \mathrm{~nm}$ ) because they must be deep enough to produce an adequate fluorescence signal and it is difficult to cut acrylic resins thinner than $70 \mathrm{~nm}$. Although thick sections obscure ultrastructure in transmission electron microscopy, in scanning electron microscopy, low accelerating voltages can be used so that only the top $30 \mathrm{~nm}$ reflect electrons $\mathrm{s}^{23}$.

In contrast, the images from the scanning electron microscope are not as crisp as those from a transmission electron microscope. Transmission electron microscopes can achieve a resolution of less than $1 \mathrm{~nm}$. However, the beam diameter for a scanning electron microscope is $4.5 \mathrm{~nm}$ when it is operated at $5 \mathrm{keV}^{23}$. Second, the production of back-scattered electrons requires stains with high atomic number such as osmium and uranium, but such staining quenches fluorescence and could not be used in our protocols before fluorescence imaging. Staining with uranyl acetate after fluorescence imaging enhances the membrane contrast considerably but still does not produce crisp images of internal membranes such as the lipid bilayers of synaptic vesicles. One potential solution would be to apply electron tomography, which might compensate for the poor staining.

Correlative fluorescence electron microscopy results in an improvement of sensitivity over immuno-EM. In many cases antibodies that work on plastic sections are not available. Although antibodies can penetrate somewhat into resins such as LR White, access to antigens remains limited in immuno-EM ${ }^{5}$. As correlative microscopy does not rely on immunocytochemistry, antibody availability is not a concern, and fluorescent proteins deep in the plastic section can be localized. In contrast, not all proteins tolerate fluorescent protein tags. In this case, if antibodies are available, they can be used for nanoscopic imaging of sections and could even be adapted for array tomography ${ }^{11}$. Despite the sensitivity of correlative fluorescence electron microscopy, at this point the method is not quantitative. Under our conditions, 30\% of the fluorescence was lost owing to the oxidizing fixatives required for membrane preservation. Truly quantitative methods await the development of more robust fluorescent proteins.

The resolution of a conventional fluorescence microscope is limited to $200 \mathrm{~nm}$. In practice, multiple fluorescence sources scatter the signal at high magnification into a large and blurry blob. Under optimal conditions, STED microscopy or PALM resolve a fluorescent source to $30 \mathrm{~nm}$ or $20 \mathrm{~nm}^{3,5}$, respectively. At these resolutions we can localize proteins to substructures of organelles in two dimensions. Correlative microscopy can also achieve higher axial resolution than current methods in nanoscopy. Z-dimension resolution in fluorescence microscopy is $700 \mathrm{~nm}, 2-3$ times worse than in the $x-y$ dimensions ${ }^{24}$. Axial resolution can be improved by using two objective lenses in the $4 \mathrm{Pi}$ microscope ${ }^{23}$, isometric STED microscopy ${ }^{25}$ and interferometric PALM ${ }^{26}$, but these arrangements have their own limitations. By sectioning the tissue into 70-nm serial sections, subdiffraction resolution is imposed on the signal simply by section thickness. Super-resolution in three dimensions can be achieved by reconstructing the volume of the tissue ${ }^{13,27}$.

STED microscopy and PALM each have advantages and disadvantages relative to sensitivity and resolution, and a choice must be made depending on the application. STED microscopy is robust because Citrine is bright, and this fluorophore survives sample preparation well. STED microscopy is preferable if protein levels are low and as a consequence the signal is highly sensitive to oxidation. We observed that PALM generates weaker signals because sensitivity of the photoconvertible fluorophoress to oxidation and higher background fluorescence than STED microscopy. The background signals in PALM, however, can be reduced by prebleaching the sample with intensive 561-nm laser and imposing a threshold for the emission period. PALM provided high-resolution fluorescence signals and will be arguably more useful when imaging small and crowded structures like those within the synapse.

\section{METHODS}

Methods and any associated references are available in the online version of the paper at http://www.nature.com/naturemethods/.

Note: Supplementary information is available on the Nature Methods website.

\section{ACKNOWLEDGMENTS}

We thank H. Hess and E. Betzig (Janelia Farm) for access to the PALM microscope for proof-of-principle experiments; R. Fetter for sharing protocols, reagents and 
encouragement; M. Davidson (Florida State University), G. Seydoux (Johns Hopkins Univeristy), S. Eimer (European Neuroscience Institute), R. Leube (Universität Aachen), K. Nehrke (University of Rochester), C. Frøkjær-Jensen (Utah), A. Ada-Nguema (Utah) and M. Hammarlund (Yale University) for DNA constructs. We thank Marine Biological Laboratory for equipment and funding for pilot experiments and Carl Zeiss Inc. for providing access to a beta version of the PAL-M. This research was supported by the US National Institutes of Health (NS034307), National Science Foundation (0920069) and Marine Biology Laboratory fellowship. (The Dart Neuroscience Scholars Program in Learning and Memory).

\section{AUTHOR CONTRIBUTIONS}

S.W. and E.M.J. conceived and designed experiments. G.H., R.J.H. and M.W.D. provided strains and advice. S.W. optimized the methods, prepared the samples and performed PALM imaging. A.P. and K.I.W. performed STED imaging. S.W., S.W.H. and E.M.J. wrote the manuscript. S.W.H. and E.M.J. provided funding.

COMPETING FINANCIAL INTERESTS

The authors declare no competing financial interests.

Published online at http://www.nature.com/naturemethods/. Reprints and permissions information is available online at http://npg.nature. com/reprintsandpermissions/.

1. Cox, G. \& Sheppard, C.J. Practical limits of resolution in confocal and non-linear microscopy. Microsc. Res. Tech. 63, 18-22 (2004).

2. Hell, S.W. Far-field optical nanoscopy. Science 316, 1153-1158 (2007).

3. Hell, S.W. \& Wichmann, J. Breaking the diffraction resolution limit by stimulated emission: stimulated-emission-depletion fluorescence microscopy. Opt. Lett. 19, 780-782 (1994).

4. Klar, T.A., Jakobs, S., Dyba, M., Egner, A. \& Hell, S.W. Fluorescence microscopy with diffraction resolution barrier broken by stimulated emission. Proc. Natl. Acad. Sci. USA 97, 8206-8210 (2000).

5. Betzig, E. et al. Imaging intracellular fluorescent proteins at nanometer resolution. Science 313, 1642-1645 (2006).

6. Rust, M.J., Bates, M. \& Zhuang, X. Sub-diffraction-limit imaging by stochastic optical reconstruction microscopy (STORM). Nat. Methods 3, 793-795 (2006).

7. Hess, S.T., Girirajan, T.P.K. \& Mason, M.D. Ultra-high resolution imaging by fluorescence photoactivation localization microscopy. Biophys. J. 91, 4258-4272 (2006)

8. Roth, J., Bendayan, M., Carlemalm, E., Villiger, W. \& Garavito, M. Enhancement of structural preservation and immunocytochemical staining in low temperature embedded pancreatic tissue. J. Histochem. Cytochem. 29, 663-671 (1981).

9. Rostaing, P., Weimer, R.M., Jorgensen, E.M., Triller, A. \& Bessereau, J. Preservation of immunoreactivity and fine structure of adult $C$. elegans tissues using high-pressure freezing. J. Histochem. Cytochem. 52, 1-12 (2004).
10. Morphew, M.K. 3D immunolocalization with plastic sections. Methods Cell Biol. 79, 493-513 (2007).

11. Murphy, R.M. et al. Size and structure of antigen-antibody complexes. Electron microscopy and light scattering studies. Biophys. J. 54, 45-56 (1988).

12. Sims, P.A. \& Hardin, J.D. Fluorescence-integrated transmission electron microscopy images: integrating fluorescence microscopy with transmission electron microscopy. Methods Mol. Biol. 369, 291-308 (2007).

13. Micheva, K. \& Smith, S. Array tomography: a new tool for imaging the molecular architecture and ultrastructure of neural circuits. Neuron 55, 25-36 (2007).

14. Tsien, R. The green fluorescent protein. Annu. Rev. Biochem. 67, 509-544 (1998).

15. Mello, C.C., Kramer, J.M., Stinchcomb, D. \& Ambros, V. Efficient gene transfer in C. elegans: extrachromosomal maintenance and integration of transforming sequences. EMBO J. 10, 3959-3970 (1991).

16. Frøkjaer-Jensen, C. et al. Single-copy insertion of transgenes in Caenorhabditis elegans. Nat. Genet. 40, 1375-1383 (2008).

17. Yeh, E., Kawano, T., Weimer, R.M., Bessereau, J. \& Zhen, M. Identification of genes involved in synaptogenesis using a fluorescent active zone marker in Caenorhabditis elegans. J. Neurosci. 25, 3833-3841 (2005).

18. Riemersma, J.C. Osmium tetroxide fixation of lipids for electron microscopy. A possible reaction mechanism. Biochim. Biophys. Acta 152 718-727 (1968).

19. Clancy, B. \& Cauller, L.J. Reduction of background autofluorescence in brain sections following immersion in sodium borohydride. J. Neurosci. Methods 83, 97-102 (1998).

20. Newman, G.R. \& Hobot, J.A. Resin Microscopy and On-Section Immunocytochemistry (Springer-Verlag, Berlin, 1993).

21. Thompson, R. Precise nanometer localization snalysis for individual fluorescent probes. Biophys. J. 82, 2775-2783 (2002).

22. Yguerabide, J. \& Yguerabide, E.E. Light-scattering submicroscopic particles as highly fluorescent analogs and their use as tracer labels in clinical and biological applications: II. Experimental characterization. Anal. Biochem. 262, 157-176 (1998).

23. Goldstein, J. et al. Scanning Electron Microscopy and X-Ray Microanalysis (Springer Science and Business Media, LLS, New York, 2003).

24. Hell, S.W., Lindek, S., Cremer, C. \& Stelzer, E.H.K. Measurement of the 4Pi-confocal point spread function proves $75 \mathrm{~nm}$ axial resolution. Appl. Phys. Lett. 64, 1335-1337 (1994).

25. Schmidt, R. et al. Mitochondrial cristae revealed with focused light. Nano Lett. 9, 2508-2510 (2009)

26. Shtengel, G. et al. Interferometric fluorescent super-resolution microscopy resolves 3D cellular ultrastructure. Proc. Natl. Acad. Sci. USA 106, 3125-3130 (2009).

27. Punge, A. et al. 3D reconstruction of high-resolution STED microscope images. Microsc. Res. Tech. 71, 644-650 (2008). 


\section{ONLINE METHODS}

Strains and oligonucleotides. Strains we used are listed in Supplementary Note 1. Oligonucleotides we used are listed in Supplementary Table 1.

Fluorescent labels of organelles. DNA constructs were assembled using the Multisite Gateway (Invitrogen) recombination technology adapted from bacteriophage lambda integration. DNA fragments were moved into entry clones; promoters were cloned into slot 1 clones flanked by attL4 and attR1 sites '(4-1)', coding regions are flanked by attL1 and attL2 sites '(1-2)', and 3' untranslated regions (UTRs) flanked by attR2 and attL3 sites '(2-3)'. Entry vectors can be recombined into destination vectors containing the promoter, coding region and $3^{\prime}$ UTR of choice. For histone $\mathrm{H} 2 \mathrm{~B}$, a minigene encoding Citrine with worm-optimized codons and three artificial introns (S. Eimer) was amplified by PCR using the primers oGH55 and oMPD6. This amplification introduced flanking $a t t B$ recombination sites and the resulting product was recombined with pDONR 221 (Invitrogen) using BP Clonase II (Invitrogen) to produce the attL1-attL2-containing entry clone (1-2)Citrine (pGH114). A similar strategy with primers oGH95 and EOS_rev was used to clone $t d E o s$ (M. Davidson), resulting in $(1-2)$ tdEos (pGH270).

The open reading frame (ORF) of encoding worm histone $\mathrm{H} 2 \mathrm{~B}$ (his-11) was released from the plasmid Ppie-1::GFP::HIS-11::pie-1 3'UTR (pJH4.52; G. Seydoux) by restriction digest with SpeI and ligated into the multiple cloning site in front of the unc-54 3' UTR in pMH472 (M. Hammarlund). The resulting attR2-attL3containing entry clone (pGH42) translationally fuses a histone onto the carboxy terminus of ORFs of fluorescent proteins in '(1-2)' entry clones when recombined in a Multisite Gateway LR reaction (Invitrogen). The heatshock promoter ( $\mathrm{P} h s p-16.41)$ in an attL4-attR1-containing entry clone (pCM1.57, G. Seydoux) was used to drive expression of the fluorescent protein-tagged histone $\mathrm{H} 2 \mathrm{~B}$ in an inducible manner. To enable directed insertion of the transgenes into the C. elegans genome, the recombination reactions were performed using the attR4-attR3-containing destination vector (pCFJ150; C. Frøkjær-Jensen) that includes genomic fragments flanking a Mos 1 transposon insertion ( $t$ Ti5605 II) for targeting, along with the unc-119 gene from C. briggsae for selection. Recombination of pCM1.57, pGH114, pGH42 and pCFJ150 using LR Clonase II Plus (Invitrogen) generated Phsp-16.41:: Citrine::HIS-11::unc-54 3'UTR (pGH201), and recombination of pCM1.57, pGH270, pGH42 and pCFJ150 generated Phsp-16.41:: tdEos::HIS-11::unc-54 3'UTR (pGH154).

The expression constructs were integrated using Mos1-mediated single-copy insertion (MosSCI) as described previously ${ }^{16}$. Briefly, unc-119(ed3) III mutants containing ttTi5605 II (EG4322) were injected with a mixture of plasmids containing the Mos1 transposase to mobilize the transposon, the targeting vector to provide a repair template for the resulting chromosomal break and red fluorescent markers expressed in muscles and neurons to mark extrachromosomal arrays. Offspring of the injected worms were selected 2-4 generations later for homozygous $u n c-119$ rescue and the appearance of fluorescent nuclei following heatshock. MosSCI of pGH201 generated the strain EG5582 oxSi282(Phsp16.41::Citrine::his-11::unc-54 3'UTR) II; unc-119(ed3) III and MosSCI of pGH154 generated the strain EG5576 oxSi283(Phsp16.41:: tdEos::his-11::unc-54 3'UTR) II ; unc-119(ed3) III.
For TOM20, to generate '(2-3)' entry clones that would encode a protein with fused fluorescent tags onto the $\mathrm{C}$ terminus of proteins encoded by '(1-2)' entry vectors, Citrine was amplified with oGH76 and oGH57, and tdEos was amplified with oGH96 and oGH94. These PCR products were each inserted between the attR2 and let-858 3'UTR of pADA-126 (A. Ada-Nguema) by amplifying this '(2-3)' entry vector with oGH38 and oGH39 and using In-Fusion PCR Cloning (Clontech) to produce (2-3)Citrine::let-858 3'UTR (pGH113) and (2-3)tdEos:: let-858 3'UTR (pGH271).

The sequence encoding the first 54 amino acids of the C. elegans TOM20 ortholog (tomm-20) of the outer mitochondrial membrane translocase was BP cloned to produce the '(1-2)' entry clone pMH496 (M. Hammarlund). This protein sequence is sufficient for protein targeting to the outer membrane of mitochodria ${ }^{28}$. The myo-3 promoter in a '(4-1)' entry vector was from Open Biosystems (p_K12F2.1_93). LR recombination of (4-1)Pmyo-3, pMH496, pGH113 and the destination vector pDEST R4-R3 (Invitrogen) generated Pmyo-3::TOMM-20(N-term)::Citrine:: let-858 3'UTR ( $\mathrm{pGH194),} \mathrm{and} \mathrm{recombination} \mathrm{of} \mathrm{(4-1)Pmyo-3,}$ pMH496, pGH271 and pCFJ150 produced Pmyo-3::TOMM20(N-term)::tdEos::let-858 3'UTR (pGH158).

EG5515 lin-15(n765ts) X ; oxEx1329 (Pmyo-3::TOMM-20(Nterm)::Citrine::let-858 3'UTR lin-15(+) LITMUS 38i) was made by injecting MT1642 lin-15(n765ts) with $33 \mathrm{ng} \mu \mathrm{l}^{-1}$ each of pGH194, lin-15 rescuing plasmid pL15EK ${ }^{29}$ and LITMUS $38 \mathrm{i}$ (NEB). MosSCI of pGH158 resulted in EG5998 oxSi203 (P myo3::TOMM-20(N-term)::tdEos::let-858 3'UTR unc-119(+)) II; unc-119(ed3) III.

To tag $\alpha$-liprin, the gene for the $C$. elegans ortholog (encoded by $s y d-2$ ) was amplified with oRJH19 and oRJH20 from genomic DNA. The resulting PCR product was BP cloned into pDONR 221 (Invitrogen) to generate (1-2)syd-2 (pRH247, R. Hobson). To generate '(2-3)' entry clones that would fuse fluorescent tags onto the $\mathrm{C}$ terminus of proteins encoded by '(1-2)' entry vectors, pDendra2 was amplified from Prab-3::TBA-1::Dendra2::unc-54 $3^{\prime}$ UTR (pWD264) with oRJH21 and oRJH22. This PCR product was then cloned into pGH38 as BamHI-SpeI fragment. To drive expression in the nervous system, the promoter of snt-1 was amplified with oRJH23 and oRJH24 from genomic DNA. The resulting PCR product was BP cloned into pDONR P4P1R (Invitrogen) to produce (4-1)Psnt-1 (pCFJ284; C. FrøkjærJensen). LR recombination of pCFJ284 pRH247 and pCFJ150 with either pGH113 or pWD240 resulted in Psnt-1::SYD-2::Citrine:: let-858 3'UTR (pRH409) and Psnt-1::SYD-2::Dendra2::let-858 3'UTR (pRH419).

EG6190 ttTi5605; unc-119; oxEx1490(Psnt-1::SYD-2:: citrine; unc-119(+) lin-15(+)) was made by injecting EG4322 ttTi5605; unc-119(ed3) with $25 \mathrm{ng} \mu^{-1}$ of pRH409 and $75 \mathrm{ng}$ $\mu 1^{-1}$ pL15EK. EG6191 ttTi4348; unc-119; oxEx1491(Psnt-1:: SYD-2::Dendra2; unc-119(+) lin-15(+)) was made by injecting EG5299 ttTi4348; unc-119(ed3) with $25 \mathrm{ng} \mathrm{ll}^{-1}$ of pRH409 and $75 \mathrm{ng} \mu \mathrm{l}^{-1} \mathrm{pL15EK}$. All constructs were designed using the plasmid editor APE (http://www.biology.utah.edu/jorgensen/ wayned/ape/).

Choice of fluorescent proteins. We chose Citrine for STED microscopy and tdEos or Dendra for PALM based on their characteristics and expression in C. elegans (Supplementary Note 2). 
Sample preparations for correlative microscopy. The sample preparation for electron microscopy comprised rapid freezing, acetone substitution, fixation, staining, infiltrating with plastic and polymerizing the plastic (Supplementary Note 3 ).

High-pressure freezing and freeze substitution. The fixatives and freeze-substitution media, 95\% anhydrous acetone (EMS, glass distilled) and 5\% MilliQ water were mixed in the cryogenic vials (Nalgene) (see Supplementary Note 4 for use of 5\% water) and frozen in liquid nitrogen before use. Acetone was used as the organic solvent in all fixations. In contrast to acetone, ethanol extracted membranes from neuronal tissues. This result was consistent with the idea that acetone acts as a fixative during the freeze substitution ${ }^{30}$.

The fixatives used here were $0.1-2 \%$ paraformaldehyde (EMS), 0.1-1\% glutaraldehyde (EMS), a combination of paraformaldehyde and glutaraldehyde, $0.1 \%$ acrolein (Sigma-Aldrich), 0.001-0.5\% osmium tetroxide (EMS, crystals), $0.1 \%$ potassium permanganate (EMS), and a combination of osmium tetroxide and potassium permanganate.

Worms expressing fluorescently tagged proteins were placed onto a $100-\mu \mathrm{m}$ deep, type-A specimen carrier, filled with bacteria (OP50 or HB101) and were instantaneously frozen in a BAL-TEC HPM 010 high-pressure freezer (BAL-TEC). The specimens were transferred into a cryogenic vial containing freeze-substitution media and fixatives. The cryogenic vials are then transferred into an automatic freeze-substitution unit (Leica, AFS 2), and the specimen was freeze substituted with the following program: $30 \mathrm{~h}$ at $-90^{\circ} \mathrm{C}, 5^{\circ} \mathrm{C}$ per hour to $-20^{\circ} \mathrm{C}$ and $2 \mathrm{~h}$ at $-20^{\circ} \mathrm{C}$.

Infiltration. Acetone and fixatives were completely washed out from tissues using 95\% ethanol before infiltration because the residual acetone causes improper polymerization owing to its action as a free-radical scavenger. Infiltration (30\% for $5 \mathrm{~h}, 70 \%$ for $6 \mathrm{~h}$ and $95-98 \%$ for overnight) was carried out at $-20^{\circ} \mathrm{C}$ in cryogenic vials. After the removal of fixatives, the specimens were washed with $95 \%$ ethanol six times over a period of $2 \mathrm{~h}$. Ethanol was prepared by adding 5\% MilliQ water to anhydrous ethanol (Sigma-Aldrich). The solutions for infiltration were prepared by mixing $100 \%$ stock plastic solutions with $95 \%$ ethanol in glass scintillation vials (EMS). Mixing the solutions in plastic vials will cause incomplete polymerization. We tested the resins Lowicryl K4M (EMS), LR Gold (EMS), LR White (EMS) and glycol methacrylate (GMA). The components for these resins are listed in Supplementary Note 5. The formulas for $100 \%$ stock solutions are as follows. For K4M, $17.3 \mathrm{~g}$ methacrylic and acrylic esters, $2.7 \mathrm{~g}$ triethyleneglycol-di-methacrylate, $10 \mathrm{~g}$ benzoin-methyl-ether and $5 \%$ water were mixed. LR Gold was mixed with $5 \%$ water. Ten grams of catalyst, benzoyl peroxide, was mixed in $500 \mathrm{ml}$ LR White one day before use. $\mathrm{pH}$ of LR White with 2-5\% water tended to be low $(\sim 5.5)$, which was too acidic for most fluorescent proteins. Therefore, we neutralized the $\mathrm{pH}$ using ethanolamine as follows. The catalyzed LR White was mixed with $2-5 \%$ water depending on the batch and neutralized with ethanolamine $(5 \mu \mathrm{l}$ in $20 \mathrm{ml}$ of catalyzed LR White). Fully neutralized LR White ( $\mathrm{pH}$ 7-7.4) could not be completely polymerized, and the sections were torn or destroyed upon sectioning. We determined the amount of ethanolamine compatible with full polymerization to be $0.025 \%$ ( $5 \mu \mathrm{l}$ in $20 \mathrm{ml}$ of LR White), which increased the $\mathrm{pH}$ to 6.5 . The $\mathrm{pH}$ of the catalyzed LR White dropped as the storage duration increased, and thus we only used catalyzed LR White that was less than 1 month old. For GMA, $67 \mathrm{ml}$ glycol methacrylate, $30 \mathrm{ml}$ butyl methacrylate, $3 \mathrm{ml}$ water and $0.6 \mathrm{~g}$ benzoyl peroxide were mixed and used for all infiltration steps.

Polymerization. The worms were dissociated from bacteria, which was the space filler for high-pressure freezing. Because acrylic resins do not cross-link to tissues, in particular the cuticle, the tissues needs to be surrounded by the plastic as much as possible. Otherwise the tissue can break away from the matrix and experience distortion. The worms were then embedded in a cap of a polypropylene BEEM capsule (EBSciences, TC). Polypropylene capsules were used because LR White does not polymerize completely in a polyethylene capsule. A disc of aclar film (EMS) was placed in the bottom of the BEEM capsule, prepared by 3/8-inch paper punches (Ted Pella; Disc Punches). A few worms from each condition were mounted on the glass slide before polymerization, and the fluorescence was observed on a Zeiss Axioskop with a $63 \times$ plan-Apochromat (numerical apterture $(\mathrm{NA})=1.40$ ) objective and imaged using a digital camera (Diagnostic Instruments). ImageJ was used to measure the photon intensity in each case.

For K4M and GMA polymerization, $1 \mathrm{ml}$ of K4M or GMA was mixed with $1.5 \mu \mathrm{l} N, N$-dimethyl- $p$-toluidine (Sigma-Aldrich) and dispensed into the embedding cap containing the specimen. For LR Gold polymerization, $0.1 \%$ benzoyl peroxide was mixed into LR Gold, and the catalyzed LR Gold was applied to the specimens in the embedding cap. For LR White polymerization, LR White accelerator (Ted Pella) was added at $1.5 \mu \mathrm{l}$ per $1 \mathrm{ml}$ of catalyzed LR White. The embedded specimens were filled with this mixture. In each case, the embedding caps were covered with another layer of aclar film to block oxygen and thus allow polymerization of the plastic. The polymerization was carried out for $24 \mathrm{~h}$. The polymerized blocks were stored in nitrogen-filled, vacuumed bags in the freezer at $-20^{\circ} \mathrm{C}$ if not sectioned immediately. We collected 70-500 $\mathrm{nm}$ sections on coverslips using an ultramicrotome (Leica, UC6) and imaged using a Zeiss Axioskop. For STED and PALM imaging, 70-100 nm ultrathin sections were collected from each strain mounted on the precleaned coverslips (\#1.5, $18 \mathrm{~mm} \times 18 \mathrm{~mm}$ for STED and \#1.5, $25 \mathrm{~mm}$ diameter for PALM).

Fluorescence quantification. Loss of fluorescence intensity was monitored using Image through all procedures from postinfiltration to sectioning. Using a point-selection tool, the intensity of 3-4 fluorescent spots from 2-3 worms in each condition was measured. The obtained values were averaged and compared.

Coverslip cleaning. Coverslips for PALM imaging were incubated in the Piranha solution ( 3 parts sulfuric acid: 1 part hydrogen peroxide) for an hour to reduce background fluorescence. The Piranha solution was then washed off thoroughly six times with MilliQ water. The coverslips were then sonicated for half an hour. The water was washed off again six times. The coverslips were dipped into $100 \%$ methanol to make the surface hydrophobic, which allows easy pick-up of sections. The coverslips were then air dried. 
Storage and shipment of specimens. Although fluorophores can be preserved effectively through electron microscopy preparation, we found the fluorescence to be very sensitive to storage conditions, specifically ambient air and temperature. Fluorescence is quenched if the samples are left out in the air at room temperature for a few days. We found that storing the samples at $-20^{\circ} \mathrm{C}$ in a bag that was filled with nitrogen gas and then evacuated can preserve fluorescence. Likewise, shipping the samples overseas requires similar conditions: nitrogen-filled, vacuumed and cold. Additionally, cutting sections of $100 \mathrm{~nm}$ thickness instead of $70 \mathrm{~nm}$ preserved the fluorescence more reliably during the shipment. Extra caution needs to be paid during the summer when the samples can be exposed to very high temperatures.

STED imaging. For STED, a solution of silica nanoparticles (Sicasta BlueF, $1 \mu \mathrm{m}, \mathrm{NH}_{2}$ functionalized, Micromod) was diluted to $1 / 10,000$ with MilliQ water. For LR White sections, about $10 \mu \mathrm{l}$ of this solution were applied to each coverglass. For GMA sections, the same silica nanoparticles were used but diluted to $1 / 500$. $10 \mu \mathrm{l}$ of the solution were applied to each coverslip and washed off after $5 \mathrm{~min}$ of incubation. The samples were kept in nitrogen until the water had evaporated. All coverslips were mounted onto single concave microscope slides (SailingBoat Lab Co.), where the concave depression was filled with MilliQ water. The samples were placed into a custom-designed STED microscope as previously described ${ }^{27}$. In brief, Citrine was excited at $490 \mathrm{~nm}$ by a diffraction-limited spot which was overlaid with a doughnut-shaped STED spot (at $590 \mathrm{~nm}$ ) featuring zero intensity in the center. The STED spot prevents fluorescence by instantly driving excited molecules back to the ground state, except in proximity of the doughnut center. Therefore fluorescence is reduced to a volume smaller than the diffraction limit. For focusing we used highnumerical-aperture objective lenses (1.4 NA Plan Apochromat, $100 \times$, oil or 1.3 NA Plan Apochromat, $63 \times$, glycerol; Leica). The epifluorescence was filtered via a 525/60 nm band-pass filter and detected by an avalanche photodiode. The silica nanoparticles were excited at $405 \mathrm{~nm}$ and detected with a second detector channel at $450 / 60 \mathrm{~nm}$. After STED imaging, the coverslips were removed from the microscope slides, dried and sent back to Utah for electron microscopy.

PALM imaging. The gold nanoparticles solution (MicospheresNanospheres, $100 \mathrm{~nm}$ or $250 \mathrm{~nm}$ ) was diluted to $1 / 10$ with MilliQ water, which was filtered with a $0.22 \mu \mathrm{m}$ syringe filter (Millipore). The solution was applied to the coverslips, and after $4 \mathrm{~min}$ of incubation, the solution was washed off with the filtered MilliQ water. The coverslips were placed in the coverslip holder for Zeiss PAL-M microscope (Carl Zeiss, PAL-M Prototype serial number 2701000005) equipped with a $100 \times$ plan-apochromat $(\mathrm{NA}=1.46)$ objective lens (Carl Zeiss). The vacuum grease was applied on the rim of the coverslip holder to minimize the drift. The region of the interest was located in the bright field and then prebleached using the intensive $561 \mathrm{~nm}$ laser illumination $(5 \mathrm{~mW})$ for $2-5 \mathrm{~min}$ until the autofluorescence was quenched. We acquired 10,000-20,000 frames at a rate of 20-30 frames s ${ }^{-1}$ using an Andor iXon DU-897D EMCCD camera (Andor Technology Plc) while photoconvertible $405 \mathrm{~nm}$ laser at $1-5 \mu \mathrm{W}$ and readout $561 \mathrm{~nm}$ lasers at $1-5 \mathrm{~mW}$ were applied simultaneously. The intensity of
$405 \mathrm{~nm}$ laser was set so that it only activated a few molecules in each frame. The centroid of the molecules was calculated and mapped using Zeiss Zen PAL-M program with the drift correction applied. Localization precision was calculated using the equation:

$$
\left\langle(\Delta x)^{2}\right\rangle=\frac{\left(s^{2}+a^{2} / 12\right)}{N}+\frac{8 \pi s^{4} b^{2}}{a^{2} N^{2}}
$$

where $\Delta x$ is the error in localization, $s$ is the s.d., $N$ is the number of photons collected, $a$ is the size of the pixel and $b$ is the background noise ${ }^{21}$. Generally, the brighter the signal, the better the localization because the brightest spot within a single fluorescent molecule can be refined to one pixel or only a few pixels in each fluorescence mass, whereas a dark dim spot will have many more pixels with the same intensity. Thus knowing the center of the fluorescent molecule is more precise if the signal-to-noise ratio is better. One must keep in mind that each dot in the PALM image is not actually observed fluorescence but rather a calculated location of each fluorophore; because the dot size is controlled by the user, it is important not to set the dot size below the experimentally defined resolution. Background fluorescence leads to ambiguity in the protein localization but can be removed from the final image. Emission from fluorescent proteins such as tdEos and Dendra typically lasts for $500 \mathrm{~ms}$ or less, whereas the emission from the background signals lasts longer than $500 \mathrm{~ms}$. By selecting molecules that were fluorescent for less than $500 \mathrm{~ms}$, most of the background fluorescence was removed.

Scanning electron microscopy imaging. The sections on coverslips were stained for $4 \mathrm{~min}$ with $2.5 \%$ uranyl acetate in water to improve membrane contrast. The sections were carbon coated and then imaged under high vacuum in a FEI Nova Nano scanning electron microscope. Electrons were collected using a backscatter detector (vCD, FEI). The immersion mode was applied to the field. The stage was negatively biased (the landing energy was set to $3 \mathrm{keV}$ ) to allow acceleration of back-scattered electrons toward the detector. The accelerating voltage and the beam current were set at $5 \mathrm{keV}$ and $0.11 \mathrm{nA}$, respectively. The grayscale of the image is then inverted to resemble TEM images, and thus, electron reflective structures appear black instead of white. The contrast was enhanced using Adobe Photoshop.

Alignment of fluorescence and electron micrographs. A fluorescence image and an electron micrograph of the same section were overlaid based on the silica bead or gold fiduciary markers, which appear electron dense in electron micrographs (Supplementary Figs. 2a and 4a). First, the image of fiduciary markers was aligned on the micrographs using Adobe Photoshop (Supplementary Figs. 2b and $\mathbf{4 b}$ ). Autofluorescence from the tissue owing to the UV-light illumination can be also used to refine the alignment. Based on the translation values we obtained from the fiduciary markers, the STED or PALM images were aligned to the corresponding electron micrographs (Supplementary Figs. 2c and 4c). For $\alpha$-liprin, the alignment could also be corrected at high magnification based on the perinuclear aggregation of $\alpha$-liprin in the electron micrograph and strong fluorescence from such aggregations in the fluorescent image. This was useful in the STED image because the beads 
sometimes moved and because the charging of the beads led to flare on the electron micrograph (Supplementary Fig. 2a,b).

For the merged panels, we applied a gradient transparency to the STED or PALM images using Adobe Photoshop so that the black background did not obscure the micrograph in the figures (Supplementary Figs. 2d and 4d). The transparency of black pixels was set to $20 \%$.
28. Kanaji, S., Iwahashi, J., Kida, Y., Sakaguchi, M. \& Mihara, K. Characterization of the signal that directs Tom20 to the mitochondrial outer membrane. J. Cell Biol. 151, 277-288 (2000).

29. Clark, S.G., Lu, X. \& Horvitz, H.R. The Caenorhabditis elegans locus Lin-15, a negative regulator of a tyrosine kinase dignaling pathway, encodes two different proteins. Genetics 137, 987-997 (1994).

30. Weibull, C. \& Christiansson, A. Extraction of proteins and membrane lipids during low temperature embedding of biological material for electron microscopy. J. Microsc. 142, 79-86 (1986). 